\title{
El proceso de Reducción de la Pobreza Multifuncional en Panamá a través de las prácticas internacionales de Responsabilidad Social Corporativa.
}

\section{Fernando T. Tomas de Cavia ${ }^{1 *}$}

1UDIMA- Universidad de Madrid, Doctorando en Derecho y Sociedad, Departamento de Administración y Dirección de Empresas.

*Autor para correspondencia. Email: de.cavia@hotmail.com

Recibido: 22 de enero de 2019

Aceptado: 27 de febrero de 2019

\section{Resumen}

Uno de los beneficios que aporta la Inversión Extranjera Directa (IED) cuando la misma se acompaña de prácticas y políticas de Responsabilidad Social Corporativa (RSC) es la reducción de la pobreza, especialmente cuando dichas inversiones se realizan en países en vías de desarrollo. Panamá es uno de los mayores receptores de Inversión Extranjera Directa de la región latinoamericana y, por lo tanto, debería poder utilizar los recursos, tangibles e intangibles, suficientes como para poder reconducir sus altos índices de pobreza, considerando todos los aspectos multifuncionales de esta. No obstante, en el año 2017, el índice de pobreza multifuncional de Panamá sitúa en torno al 19.1\% la población que se encuentra en dicha circunstancia, lo que representa en términos absolutos 777,752 personas y un total de 138,410 hogares. No parece que las buenas prácticas empresariales estén obteniendo los beneficios deseados en la sociedad panameña por lo que es fundamental conocer qué está causando esta carencia y cuáles son las fórmulas para reconducirla.

Palabras clave: Reducción de la Pobreza - Panamá - Inversión Extranjera Directa - Responsabilidad Social Corporativa

\footnotetext{
Abstract

One of the benefits of Direct Foreign Investment (FDI) when it is accompanied by practices and policies of Corporate Social Responsibility (CSR) is poverty alleviation, especially when such investments are made in developing countries. Panama is one of the largest recipients of Foreign Direct Investment in the Latin American region and, therefore, should be able to capture enough tangible and intangible resources to redirect its high poverty rates, considering all the multifunctional aspects of it. However, in 2017, Panama's multifunctional poverty index stands at around 19.1\% of the population, representing in absolute terms 777,752 people and a total of 138,410 households. It
} 
Invest. pens. crit. (ISSN 1812-3864)

Vol. 7, No. 1, enero- abril 2019

pp. 86-108

does not seem that good business practices are obtaining the desired benefits in Panamanian society, so it is essential to know what is causing this lack of success and what are the formulas to redirect it.

Key words: Poverty Alleviation - Panama - Foreign Direct Investment - Corporate Social Responsibility.

\section{Introducción}

Según establecen las directrices que emanan de los Objetivos del Milenio (ODM), la pobreza debe analizarse considerando la misma desde un punto de vista multifuncional, no solo desde el punto de vista de la falta de ingresos económicos. En definitiva, se trata de valorar y considerar, además de la mencionada falta de ingresos, todos aquellos aspectos que sufren los menos afortunados, como pueden ser las carencias en educación, vivienda, medioambiente, condiciones de trabajo y salud.

En el caso de la República de Panamá, el enfoque de pobreza multidimensional forma parte de la agenda global plasmada a través de los Objetivos de Desarrollo Sostenible, propuestos por los países que forman parte de las Naciones Unidas y adoptados por Panamá como pauta para el desarrollo.

Al utilizar dicho enfoque, el Índice de Pobreza Multidimensional de Panamá: Año 2017 (Gobierno de Panamá, 2017) obtiene resultados sorprendentes, toda vez que la República de Panamá goza de unos niveles de inversión y crecimiento que son envidiados por todo su entorno regional. Los resultados del informe muestran que el $19.1 \%$ de la población presenta pobreza multidimensional, lo que representó en términos absolutos 777,752 personas y un total de 138,410 hogares, en 2017. Las cifras son todavía más alarmantes si atendemos a los resultados obtenidos en las Comarcas Indígenas, las cuales alcanzan cifras más propias de países con un alto grado de deterioro en el nivel de desarrollo. En efecto, el nivel de pobreza multifuncional en las Comarcas Ngäbe Buglé ( $93.4 \%$ de población en situación de pobreza multifuncional), Comarca Guna Yala (91.4\% de población en situación de pobreza multifuncional) y Comarca Emberá (70.8\% de población en situación de pobreza multifuncional), obtiene unos valores que superan en 4.5 veces el valor del promedio nacional de la República.

El hecho de que la Inversión Extranjera Directa, cuando se acompaña de prácticas de Responsabilidad Social Corporativa, aporta muchos beneficios al país que la recibe, incluyendo la reducción de la pobreza, es algo comprobado y analizado empíricamente en todas las regiones del mundo. Siendo Panamá el primer atrayente de Inversión Extranjera Directa en Centroamérica y el primero en términos porcentuales sobre el Producto Interior Bruto de la Región Iberoamericana, algo parece no funcionar en el modelo panameño. No obstante, muchos de los habitantes de la región confían en las bonanzas de dichas prácticas, esperando que el mundo empresarial aporte a la sociedad aquello que los estados locales no parecen capaces de hacer.

El presente análisis tiene como objetivo analizar el modelo de Responsabilidad Social Corporativa llevado a cabo en la región Iberoamericana y concretamente en Panamá, con la finalidad de determinar por qué no es capaz de reducir la pobreza multifuncional, ni de aportar a la sociedad panameña aquello que sí que aporta en otras regiones del mundo. 
Invest. pens. crit. (ISSN 1812-3864)

Vol. 7, No. 1, enero- abril 2019

pp. $86-108$

\section{Revisión de la bibliografía. La Inversión Extranjera Directa como Potenciador de la Responsabilidad Social Corporativa y Catalizador para la Reducción de la Pobreza.}

Son varios los autores que propugnan que la Inversión Extranjera Directa que va acompañada de políticas, prácticas y estándares de Responsabilidad Social Corporativa es un dinamizador de las economías y una potente herramienta para la reducción de la pobreza, especialmente cuando las inversiones se realizan en países en vías de desarrollo. Analizaremos a continuación es estado del arte en esta materia, con las aportaciones más relevantes de los teóricos que han investigado en este tema. No debe extrañarlos la alta presencia de autores de origen indio o asiático, puesto que la zona oriental del planeta ha sido la mayor perceptora de Inversión Extranjera Directa y, por lo tanto, la que mayor número de estudios presenta en materia de RSC.

Afirman desde la Organización de las Naciones Unidas que "El desafío que suponen las políticas de RSC en los países en vías de desarrollo está enmarcado en la visión que en el año 2000 afloró a partir de los Objetivos de Desarrollo del Milenio, el cual consideró un mundo con menos pobreza, mejores condiciones de vida, menos hambrunas, menos enfermedades, mejor educación, mejora de la igualdad de oportunidades y un medioambiente más cuidado”. (UN, 2006: 3).

Según Kolstad \& Tondel (2002), "Cuanto mayor sea el nivel de IED, mayor será el nivel de crecimiento económico, lo que permitirá un mejor desarrollo social y otros objetivos de desarrollo" En su línea argumental, los autores propugnan que "el grado en que el progreso económico y social mejora la vida de todos los miembros de la sociedad se identifica a través de la distribución de la riqueza y reducción de la pobreza".

Carroll (1991) identifica en su modelo de Pirámide de RSC, que existen cuatro categorías de responsabilidad vinculados con la RSC, siendo la última de estas la responsabilidad filantrópica. Afirma el autor que "La filantropía abarca aquellas acciones corporativas que se realizan en respuesta a las expectativas de la sociedad de que las organizaciones empresariales se comporten como buenos ciudadanos. Esto incluye el compromiso activo en actos, programas, campañas o acciones que promuevan el bienestar humano. Ejemplos de filantropía incluyen las contribuciones empresariales en forma de recursos económicos, o tiempo ejecutivo, de recursos humanos o técnicos, hacia aspectos como puedan ser el arte, la educación, la reducción de la pobreza, la mejora de la salud o cualquier otra necesidad social".

El autor Visser (2009:481) afirma que "Las prácticas y políticas de RSC se vienen aplicando en los países en vías de desarrollo desde los inicios de la actividad económica de estos". Continúa su argumento afirmando que "Existe un poderoso argumento que identifica que la RSC en los países en vías de desarrollo está directamente vinculada con el entorno socioeconómico en el que las organizaciones empresariales operan y, sobre todo, en las prioridades de desarrollo que estas conllevan. Eso se vincula con la reducción de la pobreza, la provisión de salud, desarrollo de infraestructuras y educación". El autor continúa su argumento afirmando que "prácticas y políticas que promuevan la reducción de la pobreza y la buena gestión medioambiental, deben ser puestas en práctica por las organizaciones empresariales multinacionales, en sus inversiones en países en vías de desarrollo.

Newell (2005), argumenta que "como respuesta a las crisis económicas, sociales, medioambientales, vinculadas a la salud o industriales, que sufren, principalmente, los países en vías de desarrollo, la RSC es un modelo de respuesta por parte de las empresas. Un ejemplo de ello fue el rol que estas 
Invest. pens. crit. (ISSN 1812-3864)

Vol. 7, No. 1, enero- abril 2019

pp. 86-108

desarrollaron en el proceso de reducción de la pobreza que se creó como consecuencia de las crisis en Argentina durante los años 2001 y 2002".

En el año 2004, Hopkins, señala que "La necesidad de afrontar las cuestiones vinculadas a bajos estándares en el nivel de vida, explotación, pobreza, desempleo y la forma en la que se puede promover el desarrollo social en general ha sido, hasta la fecha, casi obligación exclusiva de los gobiernos. Claramente, estos, continuarán ostentando un rol preponderante en estas áreas, pero paulatinamente se está convirtiendo en una cuestión de asociación y colaboración entre los gobiernos, el sector privado empresarial y la sociedad civil".

En su análisis sobre los Objetivos de Desarrollo del Milenio, Valor (2009) propugna que "Se trata de determinar si las multinacionales, principalmente de las economías desarrolladas de los países del norte, pueden contribuir a los ODM a través de la RSC en los países con economías en vías de desarrollo, siendo el primer objetivo erradicar la pobreza y el hambre". Continúa su argumento afirmando que "Las organizaciones empresariales y algunos organismos de la ONU (Proyecto del Milenio) entienden que las empresas deben reducir la pobreza con la finalidad de mejorar sus negocios. La sociedad civil y la Comisión para el Desarrollo del Sector Privado entienden que las multinacionales deben reducir la pobreza porque es moralmente bueno y no como un fin para mejorar los resultados empresariales".

El patrimonio de utilizar la RSC como herramienta para la erradicación de la pobreza no es exclusivo de las grandes multinacionales. La autora Lewicka-Strzalecka (2013) identifica que "en el mundo empresarial, desde grandes multinacionales a pequeños proyectos de inversión, se dispone de unas capacidades y activos únicos y esenciales para contribuir al desarrollo sostenible de nuestro planeta y nuestras economías, jugando un rol fundamental e indispensable con gobiernos, sociedad civil y comunidades locales a la hora de solventar problemas y contingencias derivados de la actividad empresarial, así como afrontar desafíos globales como son el hambre, la pobreza, la desigualdad, el desempleo y el cambio climático".

Mientras los países desarrollados priorizan la protección de los consumidores y los negocios justos, los países en vías de desarrollo presentan como prioridad la reducción de la pobreza. Como claramente identifica Jenkins (2005:540) "vista la falta de un foco explícito en la reducción de la pobreza, la primera cuestión que debe ser respondida es si la RSC, tal y como se practica en la actualidad, ayuda indirectamente a dicha reducción".

Akwaowo \& Swanson (2016) afirman que "Las multinacionales, a través de su IED deben enfrentar la importante decisión sobre cómo actuar de acuerdo con las directrices de la RSC, para, entre otras cosas, reducir la pobreza en los países receptores de esta".

El equipo compuesto por Park et al., (2014) identifican el rol de los directivos y managers de las organizaciones empresariales, afirmando que "estos normalmente aceptan crear y ejecutar actividades transparentes y cooperativas que fomenten la sostenibilidad a largo plazo, implicándose en la reducción de la pobreza y en las responsabilidades sociales de las regiones en las que las multinacionales invierten. Reconocer la pobreza como una realidad y el hecho de que si no es controlada y reducida puede afectar al éxito futuro de sus organizaciones".

El objetivo de reducción de la pobreza se encuadra dentro de una estrategia global y multicultural. El prestigioso directivo indio Iqbal Sharma (2014) afirma que "La pobreza persistente y los problemas sociales y medioambientales son parte del modelo tradicional de los negocios internacionales. Para 
Invest. pens. crit. (ISSN 1812-3864)

Vol. 7, No. 1, enero- abril 2019

pp. $86-108$

alcanzar un desarrollo sostenible en el actual entorno globalizado, las compañías y los gobiernos deben realizar una aproximación nueva que tenga en cuenta las contingencias que derivan del antiguo modelo".

La disminución de la pobreza parece ser solamente uno de los aspectos de mejora que aporta la RSC en los países en vías de desarrollo que reciben Inversión Extranjera Directa de calidad. Como afirma Ismah (2009) "La prácticas de RSC identifican diferentes roles en su aplicación, entre los que destacan una mejoría en las relaciones e interdependencias entre la sociedad y la comunidad, un modelo de costes compartidos en el caso de degradación medioambiental, un esquema de transferencia de tecnología desde los países avanzados a aquellos en vías de desarrollo, posibilidad de reducción de la pobreza en las comunidades, mejora al respeto por los derechos humanos y facilidades a la hora de compartir bases de datos que faciliten las funciones de las organizaciones públicas".

El rol de la sociedad civil, principalmente a través de organizaciones sin ánimo de lucro, también es preponderante a la hora de accionar mecanismos de reducción de la pobreza. Tal y como afirman AlShammari \& Masri (2016) "Aspectos como el cambio climático, los negocios éticos o la reducción de la pobreza son atendidos por este tipo de organizaciones y, por lo tanto, están influyendo en las prácticas de las empresas, motivando un profundo impacto en la forma en la que los negocios son gestionados".

Los profesores Brown et al., (2004) aseguran que la IED ha jugado un importante rol en la reducción de la pobreza, así como en el incremento de las condiciones salariales y laborales en los países en vías de desarrollo. Por esa razón, muchos países emergentes han implementado nuevas políticas y reformas para propiciar la atmósfera correcta que atraiga la IED, de forma que consigan reducir la pobreza, crear oportunidades laborales, fomentar el crecimiento económico, proteger el medioambiente, crear nuevas infraestructuras, atraer innovaciones tecnológicas y políticas fiscales y de buen gobierno.

Según el Fondo Monetario Internacional, FMI (2013:6) “Con políticas y estándares de RSC, las economías locales pueden beneficiarse de las ventajas inherentes a la Inversión Extranjera Directa, entre ellas el alivio a la pobreza y una mejor redistribución de la renta”.

Por su parte, Khan (2000), estableció que "Para la reducción de la pobreza deben concurrir al menos dos condiciones: (i) debe existir crecimiento económico con una base sostenible y continuada y (ii) el crecimiento económico debe ser neutral con respecto a la redistribución de la renta, reduciendo las desigualdades". También Ravallion \& Datt (1999) afirman que "La pobreza persistente de una parte sustancial de la población mitiga las capacidades de crecimiento económico. En adición a lo anterior, la reducción de la pobreza presenta una mejor gestión en el caso de que las terceras partes interesadas o stakeholders de las organizaciones empresariales se vinculen con esta y formen parte del proceso".

Una de las más destacadas aportaciones al tema la realizan Rossi \& Dupont (2016) cuando afirman que "La filantropía se configura como una herramienta que puede utilizarse para la reducción de la pobreza y la obtención de objetivos medioambientales. Por ejemplo, los actuales programas de financiación y fomento de las energías renovables y la utilización de bio-fuel pueden contribuir a la creación de puestos de trabajo y el fomento del empresariado local, lo que contribuye a el incremento de la calidad de vida y la reducción de la pobreza en los países en vías de desarrollo. Los fondos empleados en ello deben quedar sometidos a rigurosos controles y modelos de reporte que faciliten el seguimiento de su uso correcto. Son las organizaciones no gubernamentales y algunas organizaciones caritativas las que, juntamente con las empresas multinacionales y sus programas de Responsabilidad 
Invest. pens. crit. (ISSN 1812-3864)

Vol. 7, No. 1, enero- abril 2019

pp. 86-108

Social Corporativa, facilitan dichos fondos y contribuyen a la reducción de la pobreza, siendo los miembros de las comunidades locales, los beneficiarios de estos programas". Continúan su argumento manifestando que "La forma en la que los gobiernos utilizan sus presupuestos para dotar de recursos a las políticas de reducción de la pobreza, el compromiso de las compañías multinacionales con dichos objetivos, a través de sus políticas y prácticas de Responsabilidad Social Corporativa, la consideración de los intereses de todas los stakeholders, modelos de control, transparencia, responsabilidad y cumplimiento, mecanismos de buen gobierno corporativo, modelos efectivos de comunicación que eliminen asimetrías de la misma, así como la restructuración de los modelos de funcionamiento de las filiales que las organizaciones empresariales multinacionales disponen en países en vías de desarrollo, sin duda contribuirán a la reducción de la pobreza, facilitarán un medioambiente limpio y descontaminado, lo que incrementará la calidad de vida de las actuales y futuras generaciones".

Lamentablemente, algunas prácticas corporativas como son: políticas de precios de transferencia, evasión de impuestos, abuso de poder en el mercado, reducción de la pobreza o fomento de la igualdad no son, en muchas ocasiones, parte de las agendas de las organizaciones empresariales multinacionales en sus políticas y actividades de inversión extranjera directa. Esta afirmación es confirmada por autores como Henderson (2001), Branco y Rodrigues (2007), o Amaeshi et al (2006).

\section{La pobreza multidimensional en Panamá}

El concepto de pobreza no solo puede suscribirse a la falta de recursos económicos o materiales debiendo considerar todos sus aspectos multidimensionales. Su medición tradicional se ha basado en el cálculo de la fuente de ingresos como fórmula para mediar la capacidad de adquirir bienes y servicios, pero este modelo olvida varios aspectos que inciden en la pobreza de los pueblos, como son el acceso a la educación, salud, empleo o vivienda. Es por esta razón que la pobreza requiere de una fórmula de medición "multidimensional" que permita abarcar elementos que nos ofrezcan un mejor entendimiento de lo que significa ser pobre. Aflora así la necesidad de disponer de un índice que capture con mayor detalle el nivel de privaciones que experimentan aquellos que viven en condiciones de pobreza, por lo que surge el Índice de Pobreza Multidimensional (IPM).

Según se afirma en el informe denominado Índice de Pobreza Multidimensional de Panamá: Año 2017 (Gobierno de Panamá, 2017) "en la actualidad el enfoque de pobreza multidimensional forma parte de la agenda global plasmada a través de los Objetivos de Desarrollo Sostenible (ODS), propuestos por los países que forman parte de las Naciones Unidas y adoptados por Panamá como pauta para el desarrollo". Los ODS establecen como su primer objetivo "poner fin a la pobreza en todas sus formas en todo el mundo", teniendo como meta específica "de aquí a 2030, reducir al menos a la mitad la proporción de hombres, mujeres y niños de todas las edades que viven en la pobreza en todas sus dimensiones con arreglo a las definiciones nacionales".

Continúa el informe afirmando que "El concepto de pobreza multidimensional toma en consideración de modo integral las múltiples privaciones y carencias que experimentan simultáneamente los individuos y hogares en múltiples dimensiones del bienestar distintas al ingreso, tales como salud, educación, trabajo, medio ambiente, nivel de vida, entre otros. El IPM representa una forma de medir estas privaciones y carencias de las personas, aportando una visión integradora de la situación y revelando el nivel de pobreza multidimensional en un país. La información que arroja el IPM puede ser desagregada por área geográfica, subgrupos de personas o carencias específicas. Por ejemplo, una persona puede estar en condiciones de pobreza multidimensional por no tener acceso a atención 
Invest. pens. crit. (ISSN 1812-3864)

Vol. 7, No. 1, enero- abril 2019

pp. $86-108$

médica, mala nutrición y falta de agua potable, mientras que otra puede estarlo por experimentar carencias de electricidad, precariedad de la vivienda, desempleo o poca escolaridad. De allí el enfoque de la pobreza como es un fenómeno multidimensional".

Los indicadores del IPM utilizados para el estudio realizado en Panamá para el año 2018, identifican las siguientes dimensiones:

- Educación: Identificando la falta de asistencia a la escuela, la repetición de curso, una o más veces dentro de un mismo año escolar o un resultado educativo ineficiente.

- Vivienda, servicios básicos y acceso a internet: Que se basa en la precariedad de los materiales utilizados para la construcción de la vivienda, el nivel de hacinamiento por habitación, la carencia de electricidad o la falta de acceso a internet.

- Ambiente, entorno y saneamiento: Que resalta la afección de los hogares como consecuencia de fenómenos naturales, la precariedad en el acceso por vías de comunicación terrestres, la inadecuada gestión de los residuos o la carencia en las infraestructuras de saneamiento de aguas residuales.

- Trabajo: Que analiza el nivel de desempleo en el núcleo familiar, el nivel de precariedad en el empleo y el sometimiento laboral debido a incumplimientos en los derechos laborales básicos.

- Salud: Ponderando el acceso a los servicios públicos o privados de salud, el control del embarazo o la carencia y disponibilidad de agua potable.

Los resultados que se obtienen del Índice de Pobreza Multidimensional de Panamá: Año 2017, son los siguientes:

1) Una primera aproximación que analiza la distribución geográfica de la pobreza en Panamá obtiene como resultados los siguientes:

a) Los resultados para el año 2017 indicaron que el porcentaje de personas en condición de pobreza multidimensional se ubicó en el 19.1\%, lo que representó en términos absolutos 777,752 personas. En cuanto a hogares, se encontraron en condición de pobreza multidimensional el 12.2\% de estos, lo equivalente a 138,410 hogares.

b) En las comarcas indígenas fue en donde se presentaron las mayores proporciones de personas pobres multidimensionales, siendo el nivel de incidencia el siguiente: Comarca Ngäbe Buglé (93.4\%), Comarca Guna Yala (91.4\%) y Comarca Emberá (70.8\%). El promedio de estos tres valores supera en 4.5 veces el valor del promedio nacional.

c) En cuanto a las provincias, las tres con mayor porcentaje de personas en condición de pobreza multidimensional y por encima del promedio nacional, fueron: Bocas del Toro (44.6\%), Darién $(40.0 \%)$ y Coclé $(22.6 \%)$. En las dos primeras habitan una amplia población indígena, $62.6 \%$ y $31.1 \%$ respectivamente, Mientras en el resto tienen menores proporciones: Los Santos (4.2\%), Herrera $(7.2 \%)$ y Panamá $(8.5 \%)$.

d) Una comarca indígena y dos provincias concentran el $53.7 \%$ de las personas en pobreza multidimensionalmente (417,851 personas), estas son: la comarca Ngäbe Buglé $(24.6 \%$ o 191,634) y las provincias de Panamá $(17.1 \%$ o 133,237) y Panamá Oeste (12.0\% o 92,980). Esta comarca es la que mayores niveles de pobreza ostenta, mientras que en las provincias la misma se sitúa por debajo del $16.0 \%$. Por el contrario, en una comarca indígena y tres 
provincias se encuentra el 5.5\% o 42,581 personas privadas: Darién $(2.8 \%$ o 21,606), comarca Emberá $(1.1 \%$ u 8,603), Herrera $(1.1 \%$ u 8,456) y Los Santos $(0.5 \%$ u 3,916). En esta comarca aproximadamente 7 de cada 10 personas y en Darién 4 de cada 10 están en pobreza multidimensional, mientras que en Herrera y Los Santos esta relación es apenas de 7 y 4 de cada 10 personas.

e) Las personas multidimensionalmente pobres experimentaron su condición con una intensidad de $43.5 \%$ a nivel nacional. En otras palabras, en promedio los pobres multidimensionales tienen carencias en 8 o más indicadores simultáneamente dependiendo de la dimensión en que se encuentren.

f) Teniendo en cuenta que la población específica de estudio son las personas en condición de pobreza multidimensional, al desagregar los resultados por áreas geográficas, podemos observar que el nivel de carencias que experimentan estas personas en cualquier parte del país es un poco similar, pero a la vez relativamente alto. Es así como, en las comarcas indígenas Ngäbe Buglé y Güna Yala fue en donde el nivel del promedio de carencias fue mayor, la intensidad de la pobreza en cada una fue de: 55.0\% y 49.7\%, respectivamente. Es decir, que en promedio las personas en condición de pobreza multidimensional en estas comarcas están privados alrededor de la mitad de los indicadores que conforman el índice para Panamá. En el caso de la tasa de intensidad de la Comarca Emberá (42.2\%), estuvo muy por debajo de la presentada por las dos comarcas anteriores e incluso fue menor a la del promedio nacional.

g) En el caso de la provincia de Bocas del Toro, que fue la que presentó la mayor tasa de incidencia de pobreza, también concurrió con el más alto porcentaje de intensidad (42.3\%). Pero no fue así en el caso de la segunda y tercera provincia con mayor incidencia (Darién y Coclé), seguidas por las provincias de Veraguas y Colón, con un promedio de carencias de $42.0 \%$ y $39.9 \%$, respectivamente. Lo que evidencia también, en contra parte con lo sucedido con la provincia de Bocas del Toro, que no necesariamente en donde hay una mayor cantidad de personas en condición de pobreza es en donde esas mismas personas presentan el mayor número de carencias en promedio.

h) Al realizar el análisis por regiones, las comarcas indígenas fueron las que presentaron los mayores Índices de Pobreza Multidimensional. En el caso de la Ngäbe Buglé el índice IPM se ubicó en 0.514. Entre los principales factores que contribuyeron a que las personas multidimensionalmente pobres de esta comarca vivan en esta condición estuvieron: logro educativo insuficiente (11.4\%), precariedad del empleo (10.1\%), manejo inadecuado de los residuos $(8.4 \%)$, desempleo y trabajo familiar sin remuneración $(7.7 \%)$, y precariedad de los materiales de la vivienda $(7.5 \%)$.

i) En la comarca Güna Yala resultó un índice IPM de 0.455. También fue común en esta comarca que el indicador de logro educativo insuficiente $(12.2 \%)$ y la precariedad del empleo $(11.1 \%)$ fueron los dos indicadores con la mayor contribución a la pobreza multidimensional. No obstante, a diferencia de la comarca Ngäbe Buglé, el tercer y cuarto indicador con mayor contribución fueron: la carencia de servicio sanitario (10.1\%), hacinamiento $(9.6 \%)$, seguido del manejo inadecuado de los desechos $(9.4 \%)$.

j) En la Comarca Emberá el índice IPM fue de 0.299. La contribución a este índice presentó una configuración similar a la de Ngäbe Buglé, pero no siguiendo el mismo orden o porcentaje de contribución, con la excepción de los indicadores de desempleo y trabajador familiar; este último resultando como quinto indicador con mayor contribución a la pobreza multidimensional fue repitencia escolar (8.4\%). 
Invest. pens. crit. (ISSN 1812-3864)

Vol. 7, No. 1, enero- abril 2019

pp. $86-108$

k) Siguiendo este mismo orden, las provincias con los mayores índices de pobreza multidimensional fueron: Bocas del Toro $(18.9 \%)$, Darién $(15.9 \%)$, Coclé $(8.7 \%)$, Veraguas $(8.0 \%)$ y Colón $(6.6 \%)$. En general, entre todas las divisiones administrativas fueron comunes los indicadores con mayor contribución al IPM. Sin embargo, dentro de estas 5 provincias con mayores niveles de pobreza multidimensional, otros indicadores también presentaron contribuciones altas. Por ejemplo, en el caso de Coclé como quinto indicador que contribuye a la pobreza multidimensional estuvo el acceso a las vías de comunicación terrestre $(8.2 \%)$, en Veraguas este mismo indicador ocupó el cuarto lugar en contribución (8.6\%) y la carencia de electricidad en la vivienda $(7.0 \%)$, y en Colón, como quinto indicador con mayor contribución se encontró la falta de acceso a servicios de salud (7.9\%).

2) Por lo que hace referencia a la educación, los resultados obtenidos identifican que:

a) La de educación $(23.9 \%$ ) es la magnitud que en mayor medida contribuye a la pobreza multidimensional. De los tres indicadores que miden las carencias en esta dimensión, el logro educativo insuficiente fue el de mayor contribución $(12.4 \%)$ e incluso dentro del total de indicadores.

b) El 15.3\% de las personas viven en hogares en pobreza multidimensional por este indicador, el 43.6\% correspondió al grupo de personas en condición de pobreza que debió contar como mínimo con una educación media completa, $40.2 \%$ por aquellos que no contaron con educación primaria completa y el $16.2 \%$ por los adultos mayores que no saben leer y escribir.

c) El $14.0 \%$ de niños o adolescentes, entre 6 y 17 años, que asisten a la escuela indicaron haber repetido una o más veces un año escolar. En esta condición se encontró que un $8.0 \%$ de la población que vive en hogares multidimensionalmente pobres.

d) $\mathrm{Al}$ menos un niño o adolescente, entre 4 y 17 años, no asistió a la escuela, lo que según la IPM 2017 corresponde al 6.7\%. Como resultado se obtuvo que el $6.4 \%$ de la población reside en hogares en pobreza multidimensional por este indicador.

3) Atendiendo a la vivienda, servicios básicos y acceso a internet, el estudio identifica que:

a) El indicador con mayor contribución dentro de la dimensión fue sin uso de internet, arrojando que un $5.9 \%$ y de las personas multidimensionalmente pobres el $9.8 \%$ viven en hogares que no tuvieron acceso al uso del internet en los últimos seis meses.

b) El segundo indicador con mayor contribución dentro de esta dimensión fue el de la precariedad de los materiales de la vivienda. Un 8.5\% de las personas multidimensionalmente pobres residían en viviendas que no contaban con los materiales adecuados, dependiendo del área o de las características propias del entorno que inciden en el tipo de vivienda utilizada.

c) Finalmente, el indicador donde la menor cantidad de personas en pobreza multidimensional $(6.3 \%)$ fue privado en esta dimensión fue carencia de electricidad en la vivienda. Se consideró carente a aquellas personas que utilizaban como fuente principal de alumbrado en la vivienda el queroseno, combustible diésel, gas, velas u otro tipo de recurso. 
Invest. pens. crit. (ISSN 1812-3864)

Vol. 7, No. 1, enero- abril 2019

pp. 86-108

4) En el apartado de ambiente, entorno y saneamiento, el estudio identifica como principales conclusiones las siguientes:

a) En Panamá, 3.3\% de la población que vive en hogares multidimensionalmente pobres, ha sido afectada por daños a su vivienda a causa de fenómenos naturales.

b) El 8.1\% de las personas residentes en hogares pobres multidimensionales, cuenta con una vía de comunicación principal en condiciones desfavorables para su libre paso durante el año, interfiriendo de este modo en el acceso de las familias a otro tipo de servicios, como pueden ser servicios médicos, económicos o escolares, entre otros.

c) Existe un $13.9 \%$ de las personas residentes en hogares considerados pobres multidimensionales, que tiene un manejo inadecuado para la eliminación de los residuos generados, tanto para el área rural como la urbana.

d) El 9.1\% de la población de hogares multidimensionalmente pobres, presenta carencias en las infraestructuras de saneamiento de aguas residuales, tanto en el área urbana como en el área rural. Esto trae como consecuencia enfermedades como la diarrea, que afecta principalmente a los niños menores de 5 años, además de problemas de malnutrición.

5) En lo que respecta a trabajo, se obtienen las siguientes conclusiones:

a) El desempleo en Panamá es un problema netamente urbano, ya que en las áreas rurales casi toda la población económicamente activa se dedica a actividades como la agricultura o la pesca, mientras que los trabajadores familiares sin pago los encontramos mayormente en las áreas rurales de Panamá. Un hogar se considera privado en el indicador desocupado y trabajador familiar sin pago si al menos una persona de 18 años o más de edad está desocupada, o es ocupada como trabajador familiar sin pago, o es potencialmente activo, entendiéndose que es parte de la población no económicamente activa, pero está disponible para trabajar. El 9.7\% de estas personas residen en hogares multidimensionalmente pobres.

b) Un hogar se considerará pobre multidimensional si al menos una persona empleada con contrato de trabajo escrito y seguro social, de 18 o más años que trabaja 40 o más horas percibe ingresos inferiores al salario mínimo. El 1.6\% de las personas residentes en hogares pobres multidimensionales, presenta dichas circunstancias.

c) Un hogar se considera privado por precariedad del empleo, si al menos una persona de 18 años y más de edad ocupado es: empleado y no tiene un contrato escrito o no paga seguro social, empleada doméstica sin seguro social, trabajador por cuenta propia sin seguro social a excepción de agricultores, gerentes, administradores y profesionales o trabajador cuenta propia en la agricultura que no tienen ingreso independiente y lo que producen es para autoconsumo. En Panamá, $14.9 \%$ de la población que vive en hogares multidimensionalmente pobres, se considera en precariedad del empleo.

6) Por último, en lo que respecta a la salud, podemos deducir las siguientes conclusiones:

a) Un hogar es carente en el acceso a servicios de salud, si al menos una persona, que haya sufrido alguna enfermedad, accidente o quebranto de salud, aunque este haya sido pasajero, no haya consultado a un especialista o en su defecto, consultó a familiares u otros miembros del hogar o a un curandero. En Panamá, 8\% de la población que vive en hogares multidimensionalmente pobres, presenta carencias por falta de acceso a servicios de salud. 
Invest. pens. crit. (ISSN 1812-3864)

Vol. 7, No. 1, enero- abril 2019

pp. $86-108$

b) Apenas un $1.6 \%$ de las mujeres en hogares multidimensionalmente pobres, no obtuvo atención prenatal durante su embarazo.

c) En Panamá, el 8.8\% de las personas residentes en hogares multidimensionalmente pobres, son carentes en la disponibilidad de agua potable, ya sea por el tipo de fuente o el escaso tiempo que el hogar cuenta con el suministro.

En el Multimensional Review of Panamá - Volume 1 y 2, publicado por la OCDE en 2017, en cuanto a la gestión de la pobreza, destacan los dos siguientes aspectos:

1- Panamá debe hallar nuevas formas de crecimiento, además, del Canal de Panamá, el sector financiero y la construcción, actividades o sectores que parecen incapaces, por sí solos, de seguir impulsando el progreso socioeconómico y la inclusión. Asimismo, muchos de quienes en los últimos años lograron escapar de la pobreza siguen siendo vulnerables y podrían volver a caer en la misma si se produjera una desaceleración económica.

2- Aunque el organismo reconoce que Panamá ha logrado mejoras socioeconómicas en las últimas décadas gracias al fuerte crecimiento económico, debería enfocarse en una agenda de reformas para convertirse en un país de altos ingresos, sostenible e inclusivo. En ese sentido, la OCDE remarca que el desarrollo inclusivo y sostenible de Panamá dependerá en gran medida de la capacidad de lograr tres objetivos principales: 1-Desarrollar mejores habilidades y empleos formales. 2-Promover la convergencia de las regiones rezagadas y reducir las disparidades territoriales mejorando el sistema impositivo. 3- Mejorar la contribución del sector privado en la financiación para el desarrollo.

\section{El modelo de Responsabilidad Social Corporativa en América Latina y El Caribe.}

Una forma de paliar la pobreza en los países en vías de desarrollo es a través de las prácticas, políticas y estándares de Responsabilidad Social Corporativa que nacen del mundo empresarial. Gran parte del debate en esta materia se centra en el estudio de casos de empresas individuales, aplicación de las mejores prácticas, así como en argumentos morales y filosóficos que pretenden fomentar la adopción de códigos de RSC por parte de las organizaciones empresariales. Este debate se origina en los países ricos del norte, pero paulatinamente gana importancia en los países en vías de desarrollo. En general existe una diferencia relevante entre los estándares y actividades de RSC en los países americanos desarrollados (USA y Canadá) y el resto de la región americana. Podríamos diferenciar cuatro estadios en el nivel de desarrollo de la RSC en las américas (Haslam, 2004):

- Corriendo: Estados Unidos y Canadá.

- Alcanzando: Países que pertenecen al entorno de la OCDE, con un nivel aceptable de desarrollo en América Latina, como son Argentina, Chile, Brasil y México.

- Caminando: El resto de los países de América del Sur.

- Estancados: Países de Centroamérica y el Caribe.

En el segundo rango de países (alcanzando) existe una actividad de RSC privada significativa, la cual incluye compañías privadas, asociaciones empresariales, gobiernos, sociedad civil y mundo universitario. Hay que destacar que la proximidad de México con Estados Unidos motiva una mayor integración entre los negocios entre ambos países que quedan amparados por la NAFTA (North American Free Trade Agreement). Asimismo, estos países se encuentran bajo el amparo de las Guías de Conducta de la OCDE para empresas multinacionales, que facilitan y promueven las prácticas y estándares de RSC. 
Invest. pens. crit. (ISSN 1812-3864)

Vol. 7, No. 1, enero- abril 2019

pp. 86-108

El resto de los países de América del Sur (caminando) presenta una diferencia significativa con respecto al primer grupo ya que sus actividades de RSC son mucho más limitadas. Entre este grupo existen grandes diferencias, ya que el nivel que alcanza Colombia, sin ser el mismo que el del primer grupo de países, es mucho más avanzado que el que puedan tener Venezuela o Bolivia. En este tercer grupo de países se observa una baja participación del sector privado y menos implicación de los gobiernos locales. No obstante, el incremento en la participación de organizaciones multilaterales (por ejemplo, el United Nations Development Programme “UNDP”) está promoviendo el Global Compact en la región. Hay que destacar tal vez las iniciativas de algunas ONGs y de algunos programas universitarios sobre RSC.

Por último, el grupo de los países de Centroamérica y el Caribe (estancados) se observan niveles de actividades y prácticas de RSC muy bajos a pesar de que en algunos de estos países existe un alto nivel de ingresos que proviene, principalmente, de actividades turísticas en general o actividades logísticas, comerciales o construcción, en el caso de Panamá. El compromiso de los gobiernos locales es prácticamente inexistente y el del sector privado destacan, por algo más de proactividad, Costa Rica y Trinidad, gracias al trabajo de asociaciones industriales. Panamá destaca por la presencia de algunas asociaciones profesionales (SUMARSE) trabajando para promover la RSC.

Por lo tanto, podemos identificar tres tendencias globales de RSC en las américas:

- El sector privado es relativamente débil y mucha de la promoción de la RSC se realiza por parte de asociaciones industriales o asociaciones privadas.

- El compromiso gubernamental con la RSC es muy débil y no se integra dentro de sus políticas globales. Hay que destacar el rol de las organizaciones multilaterales. (UNDP)

- La cobertura de los medios está bastante extendida en las economías más industrializadas. Los cursos o asignaturas de CSR están incluidos en la programación de escuelas de negocios (probablemente reflejando la norteamericanización de las escuelas de negocios en todo el hemisferio, un fenómeno que es particularmente fuerte en México). También existen ONG independientes que trabajan para avanzar en la agenda de la RSC, pero son poco numerosas y activas.

Si bien la responsabilidad social corporativa a menudo se presenta como una iniciativa voluntaria de las empresas con la finalidad de ser buenos ciudadanos corporativos, la realidad es que las empresas privadas que eligen adoptar e implementar códigos CSR están en el centro de un sistema de presiones, incentivos y defensa que involucran a muchos otros actores. Esto es particularmente cierto en América Latina y el Caribe, donde las presiones e incentivos a menudo se originan fuera del país en el que la empresa tiene sus operaciones. Diferentes actores influyen en el sistema de RSC, destacando entre ellos las empresas domésticas, la sociedad civil a través de las ONGs, sindicatos, organizaciones empresariales y, sobre todo, empresas extranjeras que a través de sus acciones de Inversión Extranjera Directa fomentan la RSC en las economías locales y en sus actores (clientes, proveedores, gobiernos). En definitiva, la RSC en América Latina se basa en un conjunto de interacciones entre diferentes sistemas locales, regionales e internacionales, entre los que destacar la influencia de los actores internacionales en los sistemas locales.

Por lo que respecta a las entidades públicas o multilaterales, la influencia los países de la OCDE del hemisferio norte (Canadá, México y Estados Unidos), así como algunos del hemisferio sur (Argentina, Brasil y Chile), han asumido el compromiso de crear "Puntos de Contacto Nacionales" (PCN) encargados de promover a nivel nacional las Directrices de la OCDE para empresas multinacionales: 
Invest. pens. crit. (ISSN 1812-3864)

Vol. 7, No. 1, enero- abril 2019

pp. $86-108$

el instrumento clave de la organización que define las responsabilidades del buen ciudadano corporativo. Dichos PNC permanecen relativamente aislados dentro de las burocracias gubernamentales, con pocos recursos para la divulgación, el diálogo con las empresas y la promoción. La pieza central del esfuerzo multilateral para promover la responsabilidad social corporativa en las Américas fue la Conferencia de las Américas sobre Responsabilidad Social Corporativa, que representó el cumplimiento de una promesa hecha en el Plan de Acción de la Cumbre de las Américas de Quebec en 2001. La Organización de los Estados Americanos (OAS), la American Agency for Cooperation and Development (IACD) y el Inter-American Development Bank (IDB) están trabajando para promover y fomentar las prácticas y estándares de RSC en la región Latinoamericana. También por parte del World Bank se han creado una serie de materiales de aprendizaje y un foro web para aclarar y fomentar la RSC en América Latina. Por último, desde las Naciones Unidas a través del United Nations Development Program (UNDP) han sido activos en Latinoamérica promoviendo la adherencia de las organizaciones empresariales locales y regionales al Global Compact, el cual debe "Servir como un marco de referencia y diálogo para estimular las mejores prácticas y lograr la convergencia en las prácticas corporativas en torno a valores compartidos universalmente" (Kell y Ruggie, 1999: 104).

En cuanto al sector privado latinoamericano, La mayoría de las empresas que ostentan un elevado nivel de actividad, tienen algunas actividades que pueden clasificarse como participación de la comunidad, o demostrar una buena ciudadanía corporativa, independientemente de si han publicado códigos o principios de ética empresarial o RSC en su sitio web. Estos proyectos incluyen un amplio espectro de actividades, desde el apoyo a eventos culturales y deportivos, desarrollo social, educación, salud y medio ambiente. Muchas de las mismas empresas que publican actividades de RSC en sus sitios web, no parecen tener códigos de RSC regularizados con principios u obligaciones específicos, que la empresa se comprometa a cumplir. Solo algunas pocas de estas empresas han publicado proyectos o recursos destinados a la RSC. También es común, en la región, la prevalencia de fundaciones corporativas a través de las cuales se coordinan la mayoría de los proyectos filantrópicos, lo que sugiere que, "las compañías latinoamericanas desean controlar cuidadosamente el cumplimiento de sus obligaciones de la RSC dentro de una organización que fortalece el reconocimiento de su marca y trabaja con su estrategia de marketing, en lugar de distribuir la filantropía a través de organizaciones de terceros" (Haslam, 2004). Según el autor, "la diferencia más significativa entre las empresas latinoamericanas y aquellas que provienen del norte del continente es que, en estas últimas, prolifera la regulación de códigos y estándares vinculados a la RSC y su información es fácilmente accesible a través de sus páginas web y esto forma parte de un estilo de información transparente sobre el gobierno corporativo, circunstancia que no se produce en Latinoamérica"

Por lo que respecta a la sociedad civil latinoamericana, esta se configura como un conglomerado de diversos tipos de organizaciones que pasan desde aquellas inspiradas por valores religiosos a las que se fundamentan en principios empresariales. Tampoco son iguales los intereses de este tipo de organizaciones, destacando las diferencias de las ONGs, independientes del mundo empresarial, de las organizaciones sectoriales o empresariales, por lo que las organizaciones verdaderamente independientes del mundo empresarial que trabajan a favor de la RSC en Iberoamérica son pocas y mayoritariamente se encuentran financiadas por fundaciones privadas y organizaciones multilaterales. Se trata de una sociedad civil "amigable" en lugar de ser "combativa" y eso se debe, sin duda, al poder que el mundo económico/político presenta en la región, por lo que la agenda de RSC en Latinoamérica queda vinculada a las organizaciones que la financian y a los intereses de estas. Estas organizaciones realizan actividades muy similares a aquellas realizadas en los países desarrollados, entre las que 
Invest. pens. crit. (ISSN 1812-3864)

Vol. 7, No. 1, enero- abril 2019

pp. 86-108

destacan la promoción de encuestas, guías de integración, mejores prácticas, construcción de redes de contacto, conferencias o grupos de trabajo, todos ellos vinculados a la RSC. Sería exagerado afirmar que el verdadero interés por la RSC en América Latina solo proviene de fuentes externas, puesto que las demandas de los consumidores exigiendo buenos ciudadanos corporativos es creciente, especialmente en los países más avanzados como México, Chile, Argentina o Brasil. En definitiva, las organizaciones de la sociedad civil, dentro de su diversidad, son actores clave en la promoción de la RSC en América Latina, siendo la financiación desde el exterior parte del impulso detrás de este crecimiento, lo que puede ser preocupante puesto que limita el debate que permite comprender sus efectos y alentarlas a actuar.

Podemos concluir afirmando que el sistema de promoción de la RSC está regularmente establecido en América Latina y el Caribe. Cuenta con el respaldo financiero de agentes externos (ej. OCDE, matrices de empresas multinacionales, etc.) y se ejecuta a través de una red de organizaciones locales de la sociedad civil, oficinas gubernamentales, instituciones académicas y empresas privadas que no ceden su gestión a terceros. No está claro que este sistema se traduzca en acciones concretaras y que, por lo tanto, sea digno de ser financiado con los escasos fondos de las organizaciones multilaterales de desarrollo. La debilidad clave del sistema de RSC en América Latina es que, este, no se somete ni a la supervisión ni a la promoción de su cumplimiento por parte de terceras partes independientes. Reconocer cómo funciona el sistema de RSC en América Latina y el Caribe es clave para entender cómo promover la adopción de códigos internos de ética y conducta y para aumentar la seriedad de ese compromiso. Convertir los compromisos de RSC en resultados es, por lo tanto, una cuestión de:

- Promover la adopción de códigos de RSC, con criterios específicos, evaluados y auditados por terceras partes independientes.

- Fortalecer agencias y ONGs independientes capaces de evaluar el cumplimiento de los códigos y estándares de RSC.

- Financiar investigaciones que alumbren la forma en la que los incumplimientos estatales afectan a la RSC en América Latina.

- Construir una metodología común para evaluar los efectos de la RSC que se aplique a nivel sectorial o de país, contribuyendo a la generación de datos agregados que permita el establecimiento de políticas específicas de esta materia.

- Fomentar que las empresas e iniciativas privadas confíen sus prácticas de RSC a terceras partes especializadas en lugar de realizar acciones vinculadas con su marca y su estrategia de marketing e imagen corporativa.

Schimidheiny (2006) cuestiona "la apropiación de una RSC importada en América Latina, donde las contingencias más acuciantes, como son la reducción de la pobreza y la defraudación fiscal no se incluyen en la conceptualización del este tipo de responsabilidad". Como contraste, De Oliveira (2006) argumenta que "Las aproximaciones locales del desarrollo de la RSC, en América Latina, pretenden responder a los muchos problemas sociales y medioambientales de la región, como son la deforestación, el desempleo, la desigualdad en los ingresos, el crimen organizado y la corrupción". También son relevantes las opiniones de Mateo et al. (2017), que afirman que "En los países Iberoamericanos, la RSC se aplica, en muchas ocasiones, por influencia de los clústeres industriales y su acción sobre el medioambiente, la responsabilidad laboral y la reducción de la pobreza." Dicho autor realiza un argumento de una forma más constructiva, afirmando que "La RSC es vista por muchos latinoamericanos como una esperanza de cambio que afronte la pobreza persistente, la degradación medioambiental, la corrupción y el estancamiento económico”. 
Invest. pens. crit. (ISSN 1812-3864)

Vol. 7, No. 1, enero- abril 2019

pp. $86-108$

Según Petry et al. (2015) "Las principales actividades de inversión social en la región Centroamericana son por orden de importancia: educación, medio ambiente, salud, empleo. En Panamá son, también por orden de importancia: Educación, Medioambiente, Cultura y Deporte, Salud, Desarrollo Económico de la Comunidad. Por lo general, las empresas seleccionan las temáticas hacia las cuales enfocan su inversión social en función de las fortalezas del negocio y las características particulares de su actividad. Lo que buscan es mitigar los impactos negativos que provocan, maximizar sus aportes a la sociedad o una combinación de las dos estrategias. (por ejemplo, una empresa con un alto consumo de agua busca compensar esta huella con programas de recuperación de cuencas hidrográficas). Las principales poblaciones atendidas por los proyectos y programas de inversión social en la región son: Las comunidades de impacto directo con la empresa, la niñez, las personas en condición de pobreza, la juventud, la adolescencia y las mujeres. Las empresas centroamericanas son conscientes del rol fundamental que tiene el sector privado en el desarrollo de un país. Señalan que la mejor aportación social que pueden realizar es la contribución y creación de empleos decentes, pagos de impuestos y provisión de bienes y servicios. Las empresas desempeñan un rol importante en la construcción y fortalecimiento de la gobernabilidad de la sociedad y deben complementar la acción pública sin reemplazar el rol del estado u otros agentes comprometidos con el desarrollo. Otro aspecto clave es una postura empresarial ética y responsable, que consideran necesaria pero no suficiente. El punto de partida debe ser el de no hacer daño a la sociedad, haciéndose cargo de los impactos negativos que generan en su entorno social, económico y ambiental".

\section{Conclusiones y recomendaciones.}

El hecho de que la Inversión Extranjera Directa, cuando va acompañada de Responsabilidad Social Corporativa, aporta una serie de ventajas a las economías de los países en vías de desarrollo, entre las que se encuentra la reducción de la pobreza, está acreditado a nivel académico y práctico. Son muchos los autores que postulan esa afirmación y muchos los gobiernos que aplican políticas e incentivos de atracción de la Inversión Extranjera Directa, conocedores de los beneficios que esta aporta al país receptor y a su economía.

Comparadas con las prácticas, políticas y estándares de Responsabilidad Social Corporativa llevadas a cabo por empresas europeas o norteamericanas, las actuaciones llevadas a cabo en la región Centroamericana parecen ser las más distantes al modelo que propugnan los países avanzados. Algunos autores califican a los países de Centroamérica y El Caribe como "estancados" en cuanto a la capacidad de comportarse como buenos ciudadanos corporativos a sus organizaciones empresariales. No obstante, la Responsabilidad Social Corporativa es vista por muchos latinoamericanos como una esperanza de cambio que afronte la pobreza persistente, la degradación medioambiental, la corrupción y el estancamiento económico.

A pesar de que en Panamá se realizan importantes esfuerzos en cuanto a la promoción de la Responsabilidad Social Corporativa por parte de empresarios, organizaciones, sociedad civil y, sobre todo, organismos multilaterales internacionales, algo parece no estar funcionando si las ratios de pobreza multidimensional se mantienen tan altos.

Panamá es el país de la región Iberoamericana que más Inversión Extranjera Directa atrae con respecto al Producto Interior Bruto. En porcentaje, la IED se situaría en torno al 9\% del PIB en términos brutos. No obstante, sus ratios de pobreza son altos, un 19.1\% de la población total, lo que representó 
Invest. pens. crit. (ISSN 1812-3864)

Vol. 7, No. 1, enero- abril 2019

pp. 86-108

en términos absolutos 777,752 personas y un total de 138,410 hogares. La situación es todavía más preocupante si nos centramos exclusivamente en las comarcas indígenas donde se presentan las mayores proporciones de personas pobres, siendo el nivel de incidencia el siguiente: Comarca Ngäbe Buglé (93.4\%), Comarca Guna Yala (91.4\%) y Comarca Emberá (70.8\%). El promedio de estos tres valores supera en 4.5 veces el valor del promedio nacional y es muy similar al de las regiones menos avanzadas del planeta.

Las causas de esas preocupantes cifras las debemos buscar en el modelo de Responsabilidad Social Corporativa que se aplica en Panamá, cuyas características resumo a continuación:

- Las organizaciones empresariales gestionan la Responsabilidad Social Corporativa bajo el régimen de su marca y como parte de su campaña de marketing, priorizando el beneficio y reconocimiento de su imagen corporativa, o la de su fundador, sobre otros aspectos.

- Normalmente no se delegan las funciones y acciones de Responsabilidad Social Corporativa a terceros especializados, se hace de forma interna en las empresas y sin la necesaria supervisión y auditoría independiente en cuanto a los términos de responsabilidad social.

- En la mayoría de las ocasiones se confunde la Responsabilidad Social Corporativa con la filantropía. Así, se promueven iniciativas de plantado de árboles, limpieza de bosques y playas, eventos deportivos o formación interna de los empleados, pero no se acometen, frecuentemente, acciones de responsabilidad que afecten a aquellos aspectos de la cadena de valor en los que la actividad empresarial se mezcla con la sociedad.

- Dichas acciones de filantropía, que algunos definimos como "filantrocapitalismo" muchas veces se acometen por tradición familiar, por valores religiosos o por imagen social. No es que esto sea intrínsicamente malo, pero no supone la maximización de la utilización de los recursos empleados en aquello que es realmente necesario, como pueda ser la reducción de la pobreza.

No debemos entender lo anterior como una crítica, más bien como una reflexión constructiva que nos permita identificar las áreas de mejora y oportunidad, de forma que la Responsabilidad Social Corporativa se integre dentro del ADN de las organizaciones empresariales y de la sociedad en su conjunto, desde los accionistas hasta el último de los trabajadores, desde el gobierno hasta la sociedad civil. Solo de esa forma las empresas locales serán capaces de alcanzar una ventaja competitiva a través de la Responsabilidad Social Corporativa al igual que sucede en los países avanzados, donde las organizaciones empresariales ocupan la mayor parte de su tiempo atendiendo las inquietudes de sus consumidores y stakeholders, en lugar que tener que hacerlo por intentar mejorar los mínimos estándares y condiciones de vida de sus ciudadanos, entre ellos, evitar la pobreza.

Las recomendaciones que emanan de los principales autores y organismos multinacionales en cuanto a un correcto ejercicio de Responsabilidad Social Corporativa configuran el modelo que debería ponerse en práctica en la Región Centroamericana y en Panamá. Estas recomendaciones consisten en las siguientes prácticas, políticas y estándares:

- Ejercicio de las Responsabilidades: Según identifica Carroll (1991), en su Teoría de la Pirámide de Responsabilidad Social Corporativa, las organizaciones empresariales deben ejercer cuatro tipos de responsabilidades:

- Responsabilidades Económicas: Históricamente, las organizaciones empresariales fueron creadas como entidades económicas diseñadas para proveer de bienes y servicios a los miembros de la sociedad. La obtención de un beneficio fue establecida 
Invest. pens. crit. (ISSN 1812-3864)

Vol. 7, No. 1, enero- abril 2019

pp. $86-108$

como el incentivo primario para los emprendedores empresariales. Antes de ser ninguna otra cosa, las organizaciones empresariales fueron las unidades económicas básicas de nuestra sociedad. Como tales, su rol principal era el de producir bienes y servicios que satisficieran las necesidades de los consumidores, al mismo tiempo que se obtenía un beneficio en el proceso. En algún momento de la historia, la idea de la obtención del beneficio se convirtió en otra de "maximización" del mismo y este ha sido el concepto prevalente desde entonces.

- Responsabilidades Legales: La sociedad no solamente espera que las organizaciones empresariales operen bajo el principio de la maximización del beneficio; al mismo tiempo se espera de ellas que cumplan con las leyes y regulaciones que emanan de las autoridades locales, estatales o supranacionales, en aquellos lugares en los que operan. Como parte del cumplimiento con el "contrato social" que existe entre las empresas y la sociedad, se espera de estas que ejerzan su actividad económica dentro del marco legal. Las responsabilidades legales reflejan una aproximación de "ética codificada" en el sentido de que se cumpla con las nociones básicas de las operaciones "justas" según lo que establecen los legisladores. Este tipo de responsabilidades se ubican en el siguiente nivel de responsabilidad, al mismo tiempo que coexisten con las responsabilidades económicas como preceptos fundamentales del sistema de libre empresa.

- Responsabilidades Éticas: A pesar de que las anteriores responsabilidades económicas y legales, por si mismas, implican normas éticas sobre comportamiento justo y responsable, las responsabilidades éticas consideran aquellas actividades y prácticas que, o bien están prohibidas, o bien son esperadas por parte de los miembros de la sociedad a pesar de que no se encuentren codificadas legalmente. Las responsabilidades éticas incluyen aquellas normas, estándares o expectativas que reflejan una preocupación, por parte de consumidores, empleados, gobiernos, sociedad civil y todas las partes interesadas, en cuanto al mantenimiento y protección de los derechos morales de las mencionadas partes interesadas, en su sentido más amplio.

- Responsabilidades Filantrópicas: La filantropía abarca aquellas acciones corporativas que se realizan en respuesta a las expectativas de la sociedad de que las organizaciones empresariales se comporten como "buenos ciudadanos". Esto incluye el compromiso activo en actos, programas, campañas o acciones que promuevan el bienestar humano. Ejemplos de filantropía incluyen las contribuciones empresariales en forma de recursos económicos, o tiempo ejecutivo, de recursos humanos o técnicos, hacia aspectos como puedan ser el arte, la educación, la reducción de la pobreza, la mejora de la salud o cualquier otra necesidad social.

Lantos (2001) opina que existen seis estadios o jerarquía en cuanto a los niveles RSC a implementar por parte de las organizaciones empresariales, añadiendo a los cuatro de Carroll, los siguientes:

- Responsabilidad altruista: Se cumplimentan las responsabilidades filantrópicas de una organización empresarial, con independencia de que las mismas reporten o no beneficios empresariales. 
- Responsabilidad estratégica: Se cumplimentan las responsabilidades filantrópicas, que al mismo tiempo benefician a los stakeholders, favoreciendo y asegurando la rentabilidad y el bienestar de la sociedad en su conjunto.

- Aplicar la RSC en la cadena de valor: Según argumenta el autor Rebelo (2011), "Las empresas pueden tener dos tipos de visiones en cuanto a la Responsabilidad Social Corporativa:

- Interna. Mapeando los eslabones de la cadena de valor que contactan con la sociedad y verificando que, en estos, se ejerce la RSC. Cada actividad primaria de la cadena de valor tiene una vinculación con la sociedad. Desde el punto de vista de compras y aprovisionamiento (evitando emisiones de Co2 y congestión), operaciones (impacto ambiental, energía, agua y residuos), logística interna (impacto del transporte y packaging inadecuado), marketing y ventas (evitando precios abusivos o anuncios engañosos) y servicio postventa (reciclaje de productos obsoletos y protección de datos, lo que hoy denominamos "economía circular"). La mejor estrategia de valor se produce cuando la empresa añade la dimensión social a todos y cada uno de los estadios vinculados a su cadena de valor.

- Externa: Mapeando el denominado "Diamante de RSC", basado en el contexto exterior de la compañía y aspectos como la competencia comercial honesta, transparencia en el reporting, seguimiento de las leyes, aceptación de regulaciones locales, disponibilidad de RRHH, acceso a universidades y centros de investigación, estructuras físicas eficientes, presencia en colectivos y clústeres, pertenencia a organizaciones sectoriales. Presenta un efecto a largo plazo.

- Tercerizar y auditar: La cesión y delegación de las acciones, políticas y estándares de Responsabilidad Social Corporativa a terceros especializados, aplicando las correspondientes medidas auditoras independientes a la concesión de ese grado de libertad, parece ser la fórmula más extendida por las organizaciones empresariales europeas y norteamericanas para el ejercicio de su rol como "buen ciudadano corporativo".

- Considerar las necesidades de las partes interesadas o stakeholders: Las teorías más recientes en el management empresarial fomentan un nuevo paradigma basado en el enfoque de los stakeholders o de base ampliada de la empresa. El nuevo paradigma propugna una narrativa del capitalismo basada en los stakeholders, creación de valor e intercambio, que pueda ser de utilidad a la sociedad en su conjunto. Es necesario sintetizar los seis aspectos fundamentales de la teoría de stakeholders:

1- Se entiende por stakeholder cualquier individuo o grupo que tenga incidencia o quede afectado o condicionado por la actividad de la empresa.

2- La dirección estratégica de la empresa debe atender la consecución de los objetivos propios de los accionistas y de todo el amplio abanico de partes interesadas, lo que facilita la pervivencia de la organización en el largo plazo.

3- El objetivo y la razón de ser de la empresa, desde el punto de vista ético (Fernández, 2007) es la maximización del bienestar de "todos" los stakeholders. 
Invest. pens. crit. (ISSN 1812-3864)

Vol. 7, No. 1, enero- abril 2019

pp. $86-108$

4- La teoría de la empresa se incardina en un contexto moral (Freeman, 1994) puesto que existe una constante y dinámica interrelación entre esta, su gestión y la dimensión moral de su actividad.

5- La empresa debe ser entendida como un conjunto de stakeholders que interactúan entre sí de manera constante y dinámica.

6- En la teoría de stakeholders, el modelo de gestión empresarial facilita que la dimensión ética encuentre acomodo de forma natural.

- Fomento del buen gobierno corporativo: Las empresas con altos estándares de gobierno corporativo no excluyen sus inversiones en países con pobres estándares de gobierno país, más bien las fomentan. Una posible explicación de lo anterior es que la elección de localización, en países con regulaciones pobres, solo puede ser exitosa para los stakeholder de la empresa cuando los estándares de buen gobierno de la empresa son altos. Un ejercicio ejemplar de buen gobierno corporativo se sustenta en la creación y aplicación de códigos de ética y conducta de obligado cumplimiento, en la adopción de estándares contables IFRS que minimizan las asimetrías de información y fomentan la transparencia, implementación de ISO 26000, o la adopción de estándares internacionales como puedan ser los principios SOX, Global Reporting Initiative, principios Sullivan, Mesa Redonda CAUX, OCDE Guidelines, Principios de la OIT, Directrices SYGMA, Código SA8000 o Iniciativa EHI. El cumplimiento de todos, o algunos, de estos principios y directrices configuran un buen modelo de buen gobierno corporativo.

En definitiva, las organizaciones empresariales deben actuar como un buen ciudadano. De esa forma, los empleados crean un sentido de pertenencia por el desarrollo positivo que recibe la comunidad local y los stakeholders, en su conjunto, ven en la actividad empresarial una respuesta a sus demandas de sostenibilidad y compromiso con la sociedad. También se deben prevenir y, en su defecto, mitigar los daños que se produzcan en las actividades de la cadena de valor empresarial, daños pasados, presentes o futuros, lo que implica un desafío operacional que debe afrontar cada tipo de organización. Cada empresa, cada industria o cada sector pueden generar diferentes impactos de responsabilidad social en su cadena de valor. Para ello, se deben identificar objetivos claros y concretos que sean monitoreados con cierta frecuencia y de forma independiente

Para la reducción de la pobreza multidimensional no basta con la filantropía o simple entrega de dinero con objetivos más o menos definidos. No se trata de entregar caridad o simplemente "lo que sobra". Se trata de establecer las condiciones y el entorno adecuado para que la sociedad avance en su conjunto, de forma equilibrada, fomentando la educación, la salud, la vivienda, la calidad de las infraestructuras y todo ello configurado dentro de un modelo sostenible a largo plazo. Sin duda, lo anterior configura un derecho y una responsabilidad para todos.

Con sus políticas de Responsabilidad Social Corporativa, las organizaciones empresariales no deben sustituir al estado acometiendo las funciones básicas de bienestar social. Esta tarea nos corresponde a todos. El ámbito de la empresa privada, las instituciones académicas y universitarias, la sociedad civil a través de sus organizaciones sin ánimo de lucro, los gremios, sindicatos y organizaciones empresariales, el estado y, en definitiva, la sociedad en su conjunto, debemos aunar esfuerzos para facilitar la disminución de la pobreza y, de esa forma, contribuir al bienestar social y al desarrollo sostenible. Esa es la única vía para garantizar la pervivencia de las organizaciones empresariales y de nuestra sociedad en su conjunto. 
Invest. pens. crit. (ISSN 1812-3864)

Vol. 7, No. 1, enero- abril 2019

pp. 86-108

\section{Bibliografía}

AKWAOWO, E., \& SWANSON, A. (2016). Boeing Directa Investimento, Corporice Social Responsibility and poverty alleviation. Evidence from African countries. Review of Business \& Finance Studies, 7(2), 21-33. Retrieved from ftp:/ /ftp.repec.org/opt/ReDIF/RePEc/ibf/rbfstu/rbfs-v7n2-2016/RBFS-V7N2-2016-3.pdf

ALONSO-ALMEIDA, M. D. M., MARIMON, F., \& LLACH, J. (2015). Difusión de las memorias de sostenibilidad en Latinoamérica: análisis territorial y sectorial. Estudios Gerenciales, 31(135), 139-149. https://doi.org/10.1016/j.estger.2015.01.002

BJORNSKOV, C., \& MEON, P.-G. (2009). The productivity of trust. Abyhoj - Bruxelles.

BULL, B. (2004). Responsabilidad social de las empresas: ¿Una solución para el desarrollo en América Latina? (Universidad de Zuila, Ed.), Revista Venezolana de Gerencia. Maracaibo, Venezuela.

CENTENO, M. A., \& LAJOUS, A. (2018). La era de la Perplejidad. Repensar el mundo que conocimos. Retos para América Latina en el Siglo XXI. (P. Random H. G. Editorial, Ed.), BBVA-OpenMind. Madrid - España.

CORREA, M. E., FLYNN, S., \& AMIT, A. (2010). La Responsabilidad Social Corporativa en América Latina: Una visión empresarial. (División de Desarrollo Sostenible y Asentamientos Humanos, Ed.), Serie Medioambiente y Desarrollo. Santiago de Chile, Chile. Retrieved from http://pwc-interamerica.com/RSC/Informe resultados RSC-CR.pdf

DIGRABRIELE, M. (2016). Corporate Social Responsibility as a Tool for Poverty Reduction: Globalization, Corporate Social Responsibility and Poverty Revisited. In Analyzing the Relationship between Corporate Social Responsability and Foreign Direct Investment (First, pp. 22-30). Hersey PA: Business Sciende Reference (an imprint of IGI Global).

DUPONT, G., OJO, M., \& ROSSI, J. (2016). Corporate Social Responsibility and Financial Development: Promoting Global Economic Development. In Analyzing the Relationship between Corporate Social Responsability and Foreign Direct Investment (First, pp. 115-126). Hersey PA: Business Sciende Reference (an imprint of IGI Global).

DUR, A., \& SILOS, J. (2014). La RSE atrae la inversión extranjera y la internacionalización de las empresas. Informe RSE y Marca España.

FATOUROS, A. A. (2000). Seven and a half propositions on the role of the OECD guidelines outside the OECE area. In OECD PROCEEDINGS (pp. 71-81). Paris.

GALLEGHER, K. P., PORZECNSKI, R., \& LOPEZ, A. (2009). Inversión Extranjera Directa y Desarrollo Sustentable. Lecciones desde las Américas. Grupo de Trabajo Sobre Desarrollo y Medio Ambiente En Las Américas, 79. Retrieved from http://ase.tufts.edu/gdae/WGOverview.htm 
Invest. pens. crit. (ISSN 1812-3864)

Vol. 7, No. 1, enero- abril 2019

pp. $86-108$

GHALIB, A. K., \& AGUPUSI, P. (2011). How socially responsible are multinational corporations? Perspectives from the developing world. Working Paper Series N 32.

GUARDIA, D. D. LA, ARIAS, I. Z., CHINCHILLA, E. V. DE, MOREIRA, R., HUMBERT, F., \& SAIED, D. (2018). Informe-del-Índice-de-Pobreza-Multidimensional-de-Panamá-2017. Panamá.

GUTIERREZ, R., \& JONES, A. (2007). Corporate Social Responsibility in Latin America: An Overview of Its Characteristics and Effects on Local Communities. SSRN.COM, 9(May), 151187. https://doi.org/10.2298/SOC1101021I

HASLAM, P. A. (2004). The corporate social responsibility system in Latin America and the Caribbean. FOCAL Canadian Foundation for the Americas, FPP-04-1, 1-16. Retrieved from htt

HERGUEUX, J. (2016). How does Religion Bias the Allocation of Foreign Direct Investment? The Role of Institutions. Laboratoire de Recherche en Gestion \& Economie. Strasbourg. Retrieved from http://ifs.ustrasbg.fr/large/publications/publications.htm

HERZER, D., HUHNE, P., \& NUNNENKAMP, P. (2012). FDI and Income Inequality - Evidence from Latin American Economies. Kiel Institute for the Worl Economy. Kiel - Germany.

JAMALI, D. (2007). The case for strategic corporate social responsibility in developing countries. Business and Society Review, 112(1), 1-27. https://doi.org/10.1111/j.1467-8594.2007.00284.x

KOLK, A., \& VAN TULDER, R. (2010). International Business, Corporate Social Responsibility and Sustainable Development. International Business Review, 19(10), 1-14.

KOLSTAD, I., \& TONDEL, L. (2002). Social Development and Foreign Direct Investment in Developing Countries. CMI Reports. Bergen, Norway. Retrieved from www.cmi.no/public/pub2002\%0AIndexin

NICHOLS NIXON, C. L., CASTILLA DAVILA, J. A., GARCIA SANCHEZ, J., \& PESQUERA RIVERA, M. (2011). Latin america management research: Review, synthesis, and extension. Journal of Management, 37(4), 1178-1227. https://doi.org/10.1177/0149206311403151

OCDE. (2018). Multi-Dimensional Review of Panama: Volume 2: In-depth Analysis and Recommendations. OCDE Publishing. Paris. https://doi.org/https://doi.org/10.1787/9789264302549-en.

OCDE. (2017). Estudio Multi-dimensional de Panamá: Volumen 1. Evaluación inicial. OCDE Publishing, 1, 25. https://doi.org/http://dx.doi.org/10.1787/9789264222052-es

ODIA, J. O. (2006). Foreign Direct Investment, Corporate Social Responsibility and Economic Development. Exploring the relationship and Mitigating the Expectations Gaps. In Analyzing the Relationship between Corporate Social Responsability and Foreign Direct Investment (First, pp. 228251). Hersey PA: Business Sciende Reference (an imprint of IGI Global). 
Invest. pens. crit. (ISSN 1812-3864)

Vol. 7, No. 1, enero- abril 2019

pp. 86-108

OJO, M. (2016). Adressing the Imbalances of Corporate Social Responsibility in Developed and Developing Countries. In Analyzing the Relationship between Corporate Social Responsability and Foreign Direct Investment (First, pp. 253-263). Hersey PA: Business Sciende Reference (an imprint of IGI Global).

OJO, M. (2016). Analyzing the Relationship between Corporate Social Responsability and Foreign Direct Investment. (IGI Global, Ed.). Hersey PA: Business Sciende Reference (an imprint of IGI Global). Retrieved from htpp//www.igi.global.com

PATRO, C. S., \& RAGHUNATH, K. M. K. (2016). La Responsabilidad Social Corporativa como una manifestación de la Inversión Extranjera Directa. In Analyzing the Relationship between Corporate Social Responsability and Foreign Direct Investment (First, pp. 202-227). Hersey PA: Business Sciende Reference (an imprint of IGI Global).

PEREZ, P. (2018). Ocde: El desarrollo inclusivo de Panamá dependerá de mejores políticas de empleo y la reducción de la desigualdad territorial. El Capital Financiero, 4.

PETRY, I., PACHECO, L. S., MARROQUIN, D. S. M., SANCHEZ, E., LOPEZ, M., CASTILLO, H. M. DEL, \& FREIRE, C. (2015). Inversión para el Desarrollo en Centroamérica. Estudio línea base sobre filantropia e inversión social. Red Integrarse Centroamérica.

POLO, J. F. (2009). La responsabilidad social corporativa de las grandes empresas españolas con presencia en América Latina: modelos de actuación. UNIVERSIDAD COMPLUTENSE DE MADRID.

PORTER, M. E., \& KRAMER, M. R. (2006). The link Between Corporate Advantage and Corporate Social Responsibility. Harvard Business Review, 84(December), 78-92. https://doi.org/10.1287/mnsc.1090.1070

REBELO, M., \& YAO, D. (2011). The link between competitive advantage and corporate social responsibility. Strategy and Society.

RODRIGUEZ, W. K., \& ALBORNOZ, R. S. (2016). IED y desigualdad en el ingreso en Latinoamérica. Evidencia de la cointegración de datos de panel. Cuadernos de Economía, (35), $433-455$.

ROSSI, J., \& DUPONT, G. (2016). Foreign Direct Investment as a Tool for Poverty Alleviation. In Analyzing the Relationship between Corporate Social Responsability and Foreign Direct Investment (First, pp. 127-137). Hersey PA: Business Sciende Reference (an imprint of IGI Global).

RUIZ, M. A. G. (2013). Responsabilidad Social Empresarial y fiscalidad internacional en relación con la inversión directa extranjera en países en desarrollo. Journal Globalization, Competitiveness and Governability, 7(3), 34-48. https://doi.org/10.3232/GCG.2013.V7.N3.02

SCHMIDHEINY, S. (2006). A view of Corporate Citizenship in Latin America. Journal of Corporate Citizenship, (21), 17-20. https://doi.org/10.1016/j.robot.2009.06.003 
Invest. pens. crit. (ISSN 1812-3864)

Vol. 7, No. 1, enero- abril 2019

pp. $86-108$

SIKKEL, M. (2000). Review of the OECD Guidelines for Multinational Enterprises. In OECD PROCEEDINGS (pp. 65-70). Paris.

SOLANO, C. (2016). La comunicación del progreso de la Responsabilidad Social Empresarial (RSE) de las empresas panameñas que firmaron el Pacto Mundial, 4(3), 23-36.

SUANES, M. (2015). Tres Ensayos sobre Inversión Extranjera Directa y Desarrollo Económico en América Latina. UNIVERSIDAD AUTONOMA DE BARCELONA.

SUANES, M., \& ROCA, O. (2015). Inversión extranjera directa, crecimiento económico y desigualdad en América Latina. El Trimestre Económico, 82 (3) (327 julio-septiembre), 675-706. Retrieved from http://www.scielo.org.mx/scielo.php?script=sci_arttext\&pid=S2448718X2015000300675

UNCUETA, A. (2006). Responsabilidad social corporativa. Observatorio de Multinacionales En América Latina, 14, 25-42.

VALOR, C. (2009). Tres perspectivas sobre cómo pueden las empresas multinacionales contribuir efectivamente a la consecución de los ODM. Innovar, 19(33), 131-141.

VELDE, D. W. TE. (2003). Foreign Direct Investment and Income Inequality in Latin America. Overseas Development Institute, 1-64.

VISSER, W. (2009). Corporate Social Responsibility in Developing Countries. The Oxford Handbook of Corporate Social Responsibility, (October), 29.

https://doi.org/10.1093/oxfordhb/9780199211593.003.0021

VIVES, A. (2006). Social and Environmental Responsibility in Small and Medium Enterprises in Latin America. Inter-American Development Bank, Technical (Spring 2006), 39-50. https://doi.org/10.9774/GLEAF.4700.2006.sp.00006 